\title{
Risk of chronic obstructive pulmonary disease in a cohort of workers occupationally exposed to ionizing radiation over prolonged periods
}

\author{
Tamara Azizova ${ }^{1}$, Galina Zhuntova ${ }^{1}$, Evgenia Grigorieva ${ }^{1}$, Maria Moseeva ${ }^{1}$, Maria Bannikova $^{1}$, and Evgeniy Bragin ${ }^{1}$ \\ ${ }^{1}$ Southern Urals Biophysics Institute, Ozyorsk, Russia
}

\begin{abstract}
Introduction
Mayak Production Association is a Russian large-scale nuclear enterprise producing weapon-grade plutonium which was set in operation in 1948. Equipment defects and irregularity of technology process, low level of production mechanization and automatization in early years of the enterprise operation caused high levels of alpha-radioactivity in occupational air [1]. As Mayak PA workers did not use individual protection means for organs of respiratory system, alpha-particles of plutonium deposited in workers' organisms via inhalation and were accumulated in main organs of plutonium deposition (lung, liver, bones) [2]. The first plutonium-induced pulmonary fibrosis (an early tissue reaction) was diagnosed in a plutonium production plant worker in 1956 [3].

The present study was aimed to assess the risk of chronic obstructive pulmonary disease (COPD) in workers of the Mayak PA exposed to internally deposited alpha-particles.
\end{abstract}

\section{Material and methods}

The study cohort included Mayak PA workers first employed at one of the main facilities (industrial reactors, radiochemical and plutonium production plants) in 1948-1982, in total 22,377 individuals (with $25 \%$ of females).

The follow-up of the cohort started with a date when a worker was hired and lasted until the earliest of the following events: date of COPD diagnosis, date of death, 31.12.2008, date of the last available medical record for migrants and residents with unknown vital status.

By the end of the follow-up vital status data was available for $95.0 \%$ of cohort members of whom $53.5 \%$ had deceased and $46.5 \%$ were alive. Information on disease incidence through the entire follow-up period was collected for $95.0 \%$ of workers, data on smoking status was available for $97.0 \%$ of workers with quantitative parameters estimated for $51.0 \%$ of ever-smoking workers.

To perform analyses, estimates of absorbed external and internal radiation doses were provided by dosimetry system MWDS-2008 [4].

Relative risk (RR) and excess relative risk per unit dose (ERR/Gy) were calculated based on maximum likelihood using AMFIT module of EPICURE software [5]. Differences were considered as statistically significant with $\mathrm{p}<0.05$.

\section{Results}

The analysis demonstrated that COPD incidence in the Mayak PA worker cohort was significantly associated with sex, attained age, facility type, smoking status and index, calendar period and hazardous production factors which workers had been exposed to before employment at the Mayak PA (coal dust, chemicals, etc.).

The categorical analysis showed an increase of COPD risk estimates with increasing radiation doses, but significant $\mathrm{ERR}=1.30$ 95\% CI: 1.04, 1.62) was revealed only in workers exposed to internally deposited alpha-particles at lung absorbed doses exceeding $0.1 \mathrm{~Gy}$.

The table below summarizes results of ERR/Gy analysis for COPD incidence following external and internal radiation exposure.

A significant association of COPD incidence risk with dose from internally deposited alpha-particles was shown for Mayak PA workers first employed at one of the main facilities in 1948-1982.

No significant association of COPD incidence was observed with dose from external gamma-rays. The risk gained significance only after exclusion of adjustments for smoking and dose from internal alpha-radiation exposure.

To improve the accuracy of estimates of radiogenic risks for the study cohort, further studies are required to take into account characteristics of alpha-active aerosols used at the plutonium production and radiochemical plants in addition to occupational radiation doses as well as to investigate a combined effect of occupational radiation exposure and smoking.

Conclusion

The study provides evidence to increased risk of COPD in the cohort of workers occupationally exposed to internal alpha-radiation. 
Table COPD incidence risk in relation to external gamma-ray and internal alpha-particle exposures (ERR/Gy)

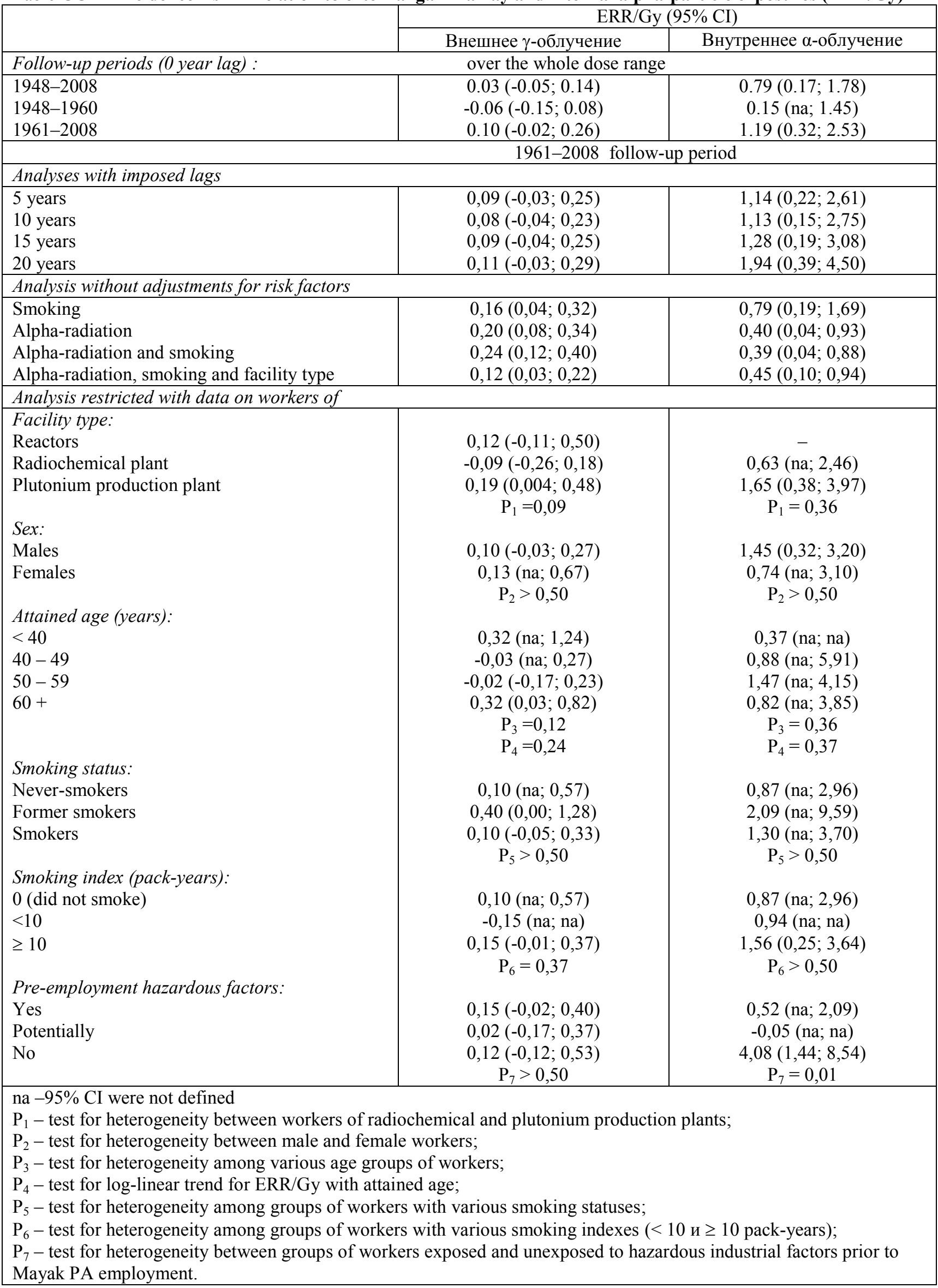




\section{References}

1. A. Kruglov, The history of the Soviet atomic industry Taylor and Francis, 2002.

2. L. Ilyin, Plutonium. Radiation Safetyt Mascow, Izdat, 2005.

3. A. Mishachev, Revisiting an issue of occupational pneumosclerosis in workers of plutoniu-239 processing facilities. Bulletin of Radiation Medicine. 1, 10-17 (1963) [in Russian]

4. V. Khokhryakov, V. Khokhryakov, K. Suslova, V. Vostrotin, V. Vvedensky, A. Sokolova, et al Mayak Worker Dosimetry System 2008 (MWDS-2008): Assessment of internal alpha-dose from measurement results of plutonium activity in urine. Health Phys 104: 366-378 (2013).

5. D. Preston, J. Lubin, D. Pierce, M. McConney, Epicure Users Guide. Hirosoft, Seattle, WA (1993). 